\title{
Physiology
}

$\begin{array}{llll}\text { Acito, A. p121 } & \text { Dick, B. p81 } & \text { Horita, S. p87 } & \text { Oliveira, J.G.G. p115 } \\ \text { Addabbo, F. p121 } & \text { Egi, M. p1 } & \text { lacobelli, S. p121 } & \text { Pande, S. p23 } \\ \begin{array}{l}\text { Almeida, M.D.V. p115 } \\ \text { Ariizumi, T. p87 }\end{array} & \text { Endo, Y. p87 } & \text { Kamijo, H. p12 } & \text { Pestana, M. p115 } \\ \text { Barasch, J. p56 } & \text { Esposito, L. p121 } & \text { Karim, Z. p126 } & \text { Procino, G. p121 } \\ \text { Bellomo, R. p1 } & \text { Fernández, B.E. p136 } & \text { Kawakami, H. p87 } & \text { Reuters, I. p95 } \\ \text { Bichara, M. p126 } & \text { Frey, B.M. p81 } & \text { Kleta, R8. p73 } & \text { Ritter, C.S. p23 } \\ \text { Bockenhauer, D. p73 } & \text { Fujita, T. p87 } & \text { Knepper, M.A. p61 } & \text { Rothstein, M. p23 } \\ \text { Bonsante, F. p121 } & \text { Gallazzini, M. p126 } & \text { Kudo, A. p87 } & \text { Sabolić, I. p107 } \\ \text { Borges, C. p115 } & \text { Garcia, F.A. p136 } & \text { Lan, D. p56 } & \text { Sampaio, S. p115 } \\ \text { Brown, A.J. p23 } & \text { Higuchi, M. p12 } & \text { Langenberg, C. p1 } & \text { Schiavi, S.C. p23 } \\ \text { Choi, M.R. p136 } & \text { Hirsh, B.J. p56 } & \text { Li, Y. p87 } & \text { Schmidt-Ott, K.M. p56 } \\ \text { Coroas, A.S.P.S. p115 } & \text { Hoffert, J.D. p61 } & \text { May, C.N. p1 } & \text { Schumacher, M. p81 } \\ \text { Correa, A.H. p136 } & \text { Hoorn, E.J. p61 } & \text { Morgera, S. p1 } & \text { Seki, G. p87 } \\ \text { del Valle Turco, V. p136 } & \text { Hora, K. p12 } & \text { Moriyama, N. p87 } & \text { Slatapolsky, E. p23 }\end{array}$

Svelto, M. p121

Tamma, G. p121

Tanaka, Y. p87

Tavares, I. p115

Valenti, G. p121

Vassiliadis, J. p23

Vogt, B. p81

Wan, L. p1

Weber, M. p95

Wiesen, K. p23

Yamada, H. p87

Yamazaki, S. p87

Yu, Z. p81

del Valle Turco, V. p136

Hoorn, E.J. p61

Moriyama, N. p87

Seki, G. p87

Slatapolsky, E. p23

\section{Subject Index Vol. 104, 2006}

Acute renal failure $\mathrm{p} 1$ Angiotensin II p136

Apoptosis p107

Aquaporin-2 excretion, preterm newborn infants p121

$\mathrm{AT}_{1}$ receptor $\mathrm{p} 136$

$\mathrm{AT}_{2}$ receptor p136

Bartter syndrome p73

Bioimpedance p115

Bioinformatics p61

Cadmium p107

Cardiac output p1

Chronic inhibition p12

- renal failure p115

Cisplatin p107

Critical illness p1

Diabetic nephropathy p12

Difference Gel Electrophoresis p61

Dopamine p136

Epithelial cell migration p95

- polarity p56

Extracellular water p115

Fibroblast growth factor-23 p23

Gas chromatography $\mathrm{p} 81$
Gene expression profiling p56

- transcription rate p126

Gitelman syndrome p73

Heavy metals p107

Hemodialysis p115

$11 \beta$-Hydroxysteroid dehydrogenase type 2 p 81

Hyperparathyrodiism p23

Hypertension p 81

Hypertonicity p126

Hypophosphatemia p23

Immunohistochemistry p87

Isolated tubules p126

Kidney p136

- development p56

- disease p23

Laminin p95

L-arginine $\mathrm{p} 12$

Lead p107

Leukemia inhibitory factor p56

$\mathrm{L}-\mathrm{N}^{\mathrm{G}}$-nitroarginine methyl esther p12

Mass spectrometry p61, p81

Matrix extracellular phosphoglycoprotein p23

Mercury p107
Mesenchymal-to-epithelial conversion p56

Monocytes/macrophages p12

$\mathrm{Na}^{+}-\mathrm{HCO}_{3}^{-}$-cotransporter p87

Nagase analbuminemic rat p81

Necrosis p107

Nephrotoxicity p107

Neutrophil gelatinase associated lipocalin p56

Nitric oxide p12

Otsuka Long-Evans Tokushima Fatty rat p12

p38 MAPK and ERK pathways p126

Parathyroid hormone p23

Pathways analysis p61

Phosphate p23

Phosphatonin p23

Preterm newborn infants, renal function $\mathrm{p} 121$

Proximal tubular cells p95

Pseudohypoaldosteronism p73

Rat kidney p87
Renal blood flow p1

- concentrating ability, preterm newborn infants p121

- Fanconi syndrome p73

- transplant p115

- transplantation p23

- vascular resistance, sepsis p1

Rho/Rho kinase pathway p95

ROMK p126

Run-off assay p126

Salt challenge p81

Secreted frizzled related protein-4 p23

Sepsis, renal vascular resistance $\mathrm{p} 1$

Septic shock pl

Sodium transport p73

Stages p56

Systems biology p61

Thick ascending limb cell culture p126

TonEBP p126

Type 2 diabetes mellitus p12

Vasopressin p61 